\title{
Clinical Analysis of the Influence of Personalized Rehabilitation Program on Fracture Patients' Rehabilitation \\ Y Liu ${ }^{1}, \mathrm{H} \mathrm{Liu}^{2}, \mathrm{Y} \mathrm{Yang}^{2}, \mathrm{~J} \mathrm{Kan}^{1}, \mathrm{~S} \mathrm{Dai}^{3}$
}

\begin{abstract}
Objective: This research analyzed the clinical influence of personalized rehabilitation program on fracture patients' rehabilitation.

Methods: Through retrospective analysis of the clinical data of 100 facture patients treated in our hospital, the patients were imposed with personalized nursing intervention. After that, a rehabilitation assessment scale was given to rate the daily activity performing ability before and after implementing personalized rehabilitation program.

Results: The result shows that after nursing intervention, patients' satisfaction on nursing and the ability of performing daily activities were both increased. Therefore, the personalized rehabilitation program can achieve more ideal effect during fracture patients' rehabilitation period.
\end{abstract}

Conclusion: To be specific, patients' awareness on fracture rehabilitation was enhanced, the movement function and self-care ability were improved in largest degree, and a higher degree of satisfaction on rehabilitation was achieved.

Keywords: Personalized Rehabilitation Program, Fracture Influence, Clinical Analysis

From: ${ }^{1}$ Linyi People's Hospital, Linyi, 276002, China, ${ }^{2}$ Affiliated Hospital of Shandong Medical College, Linyi, 276004, China, ${ }^{3}$ Qingdao Municipal Hospital, Qingdao, 266000, China.

Correspondence: Dr S Dai, Qingdao Municipal Hospital, Qingdao, 266000. China Email: yuanyangliu@163.com 


\section{INTRODUCTION}

According to relevant data analysis, it can be seen that fracture has become one of the most common injuries in our daily life. Fracture mainly refers to the interruption of integrity and continuity of bone. During the fracture treatment period, there are pretty significant particularities in following aspects: first, fracture patients need long time for bed rest. Secondly, the treatment cycle is very long. Then, most fracture patients need internal fixation operation. Finally, fixation of plaster or splint is needed (1).

Under normal conditions, fracture patients will suffer from transient dysfunction and permanent dysfunction in different degree, which will affect their daily lives. After facture, patients feel hard to handle their life by themselves, moreover corresponding psychological obstacles and mental diseases may occur, which causing severe damage to their life quality. Currently, medical staffs are mostly focusing on how to enhance patients' rehabilitation in the largest degree while preventing related complications.

Currently, most fracture rehabilitation care programs are performed according to the rehabilitation nursing principles prescribed in the text book of medical college, however, in practical situation, normally fracture patients do not like to obey such rehabilitation programs. Therefore, we should learn more successful experiences on the basis of initial stage to facilitate further improvement and deepening of optimal nursing pattern(2). This research, based on patients' practical situation and guided by evidence-based nursing principle, formulated a series of scientific and effective personalized rehabilitation programs in the hope of providing references for the treatment of fracture patients. 


\section{SUBJECTS AND METHODS}

Firstly, all patients were imposed with symptomatic treatments; after that, the nursing interventions in regular clinical nursing pathway were carried out (3). In addition, according to patients' situation, personalized nursing interventions were implemented in following steps. overall assessment was conducted once a week on admission and during hospitalization, then an overall assessment was conducted every two weeks after leaving hospital; On the basis of above operations, an overall assessment was conducted once a month to once per half year (4).

Regarding the clinical data collection, we select the method of asking to collect data including following few aspects: collect patients' basic data, and analyze the practical health condition of patients in the meantime. Not only focusing on the patients' comprehensive degree on fracture knowledge, but also analyzing patients' coping style on health problem. In addition, strictly according to the evaluation results, we should formulated a specific personalized rehabilitation care program for fracture patients, and hand a copy of the program to the patient (one copy is enough), on the other hand, we should make patients fundamentally understand their nursing program, participate and obey the care intervention arranged for them, so as to help patients and relatives to fundamentally master fracture care knowledge, fracture cause and fracture complication prevention in details(5-7).

In addition, medical staffs should master the nursing skill for on-bed patients, and master the tips in diet guide and taking medicine. Based on the practical situation of patients, a specific personalized rehabilitation nursing program should be formulated including daily activity training, environmental adaptation capacity training, and limbs functional training. 
Moreover, we should further enhance the communication between nursing staffs and patients, provide psychological intervention and help patients to adjust psychological problem, and analyzed patients' role function. On this basis, patients and their relatives can cooperate together to effectively fulfill the rehabilitation nursing program. Regarding above mentioned patients, 6 months of nursing intervention will be provided (8).

We should allocate full-time nursing staffs to implement re-evaluation rating scale so as to analyze patients' ability of performing daily activities before and after treatment, to analyze patients' limb movement function and self-care ability of daily living (9). The self-made questionnaire should be made to investigate the degree of satisfaction on the nursing program, and then according to the evaluation results, the program should be revised in time to form a better rehabilitation program.

Aiming at analyzing the clinical influence of personalized rehabilitation program on fracture patients' rehabilitation condition, this research retrospectively analyzed the clinical data of 100 fracture patients treated in our hospital. All patients agreed on this research, signed the letter of consent, and positively obeyed related nursing procedures. The $\mathrm{X}$ ray Pictures of Fracture is shown as Figure 1. The $\mathrm{X}$ ray Pictures of Fracture treatment is shown as Figure 2.

\section{RESULTS}

\section{Activity of performing daily activity}

This research analyzed patient's ability of performing daily activities before and after treatment. The result is that the daily activity performing ability before treatment was rated as 
42.32 \pm 3.81 points; while after treatment the daily activity performing ability was rated as $93.28 \pm 6.13$ points, from which we can see a significant improvement. Therefore, it shows that the personalized rehabilitation program is of significant promotion effect to fracture patient's rehabilitation.

\section{Degree of satisfaction on nursing}

Using self-made questionnaire to investigate patients' satisfaction on the personalized nursing program, it can be seen that there were 50 patients who were very satisfactory on the program, which accounted for $50.00 \%$; 20 patients who were basically satisfactory, which accounted for $20.00 \%$; 25 patients who were satisfactory, which accounted for $25.00 \%$; while only 5 patients who were not satisfactory, which accounted for $5.00 \%$. Therefore it can conclude that patients' satisfaction degree on the personalized nursing program is $95.00 \%$, which indicates that the personalized rehabilitation program plays a vital significant role in improving patients' satisfaction on fracture nursing.

\section{DISCUSSION}

With the social development, the incidence rate of fracture is getting increased, wherein the middle-aged and aged people are the sufferers which account for larger proportion. Normally after proper and scientific treatment, $90 \%$ fracture patients can recover the original body function. However due to the significant particularities in fracture treatment, patients feel difficult in realizing life self-care and also tolerate the inconvenience and pain caused by fracture for a long time (10). Due to the existence of above factors, patients and their relatives 
are bothered, in particular, some patients may bring heavy economic burden to their family. Therefore, in practical situation, medical staffed should formulated a series of scientific personalized nursing program to decrease the pain of patients, and further increase the treatment compliance of patients through implementing personalized rehabilitation program, so as to accelerate the rehabilitation process. In all, personalized rehabilitation program is of obvious clinical significance to the rehabilitation of fracture patients.

Normally, the fracture rehabilitation nursing program implemented in hospital is conducted according to the rehabilitation nursing principles proposed in the text book of medical college. On this basis, our hospital formulated a specific rehabilitation nursing program under above conditions by combining the practical clinical experiences. Due to the differences in fracture type, the age of fracture patients, the gender of fracture patients, the fracture rehabilitation can be various. The individualized variation of fracture patients will make traditional nursing method hard to meet the practical requirements of all patients in largest degree; therefore, the implementation of personalized rehabilitation program is very important in this process, which is an inevitable development trend as well.

Based on the key point of evidence-based nursing, personalized fracture rehabilitation nursing program fundamentally combines the holistic nursing and clinical nursing pathway, which can provide specific rehabilitation nursing measures according to each patient's particularity and practical situation, so as to realizing the personalization of the rehabilitation nursing measures. Based on the practical situation of each fracture patient, the hospital should formulate a specific rehabilitation nursing program to facilitate the recovery, so as to realize the complete recovery of both mental function and physiological function. 
During the implementation of personalized rehabilitation nursing, nursing staffs one hand should be equipped with abundant clinical experiences and the desire of further learning and progressing so as to master the highest level of theoretical knowledge level, on the other hand they should be proficient of using most clinical knowledge, familiar with the usage of scales and related evaluation criteria. In practical work, nursing staffs should be equipped with excellent communication ability, actively communicate with the patients, and answer patients' questions with patients. On this basis, in addition to obtaining the practical health condition of patients the health problems in deep layer should be found. Nursing staff should sort out a part of effective data before formulating the specific personalized rehabilitation nursing program. Subsequently, the program should be effectively implemented and attention should be paid to each aspect during the implementation process. Being analyzed in terms of advantages and disadvantages, the personalized rehabilitation nursing program should be further revised so as to achieve a better rehabilitation care for patients.

\section{CONCLUSION}

Through this research, it can be found that if the specific personalized rehabilitation nursing program is implemented, patients' awareness on fracture rehabilitation, body movement function can and the self-care ability of patients can be further improved. In addition, patients' satisfaction on rehabilitation care is improved, showing high rehabilitation care compliance. Therefore, the specific personalized rehabilitation program is worth of being clinically promoted 


\section{REFERENCES}

1. Min D, Jiangbo A, Hongxian F, Bin Z, Lang S. The influence of personalized rehabilitation exercise prescription on the functional recovery of hip joint after hip fracture. J China's Tissue Engineering Research and Clinical Rehabilitation 2010; 48: 9082-92.

2. Caixia L, Siqin C, Yaqin L. Influence of personalized health education prescription on the fracture rehabilitation effect of empty-nest elderly. J Modern Hospital; 2013; 2: 153-9.

3. Xiaoxia C, Jingyun Z, Yanying H. Evaluation of the effect of personalized functional rehabilitation care for patients with lower limb fracture. J Chinese and Foreign Medical Research 2013; 14: 81-9.

4. Zhenjiang Z, Bin Z, Xin W. Effect research of personalized rehabilitation treatment of elbow function on distal humeral fractures of adult patients. J Health Care Today 2015; 2: 122-32.

5. Yu Z. Clinical research of postoperative exercise rehabilitation of malleolar fracture. J Nanchang University 2012; 1: 210-7.

6. Yu Z, Min D, Hongxian F, Jiangbo A, Lang S, Zhimin P. postoperative rehabilitation of ankle joint function after ankle fracture. J Practical Clinical Med 2012; 4: 42-9.

7. Jiangbo A. Clinical application of exercise rehabilitation safety assessment in fracture of tibial plateau. J Nanchang University 2010; 10: 35-45.

8. Yuebing R, Limin Y, Chengshao Z, Luping L, Xueren T. Application of isokinetic training in postoperative early rehabilitation for elbow fracture. $\mathbf{J}$ Chin $\mathbf{J}$ 
Rehabilitation Medicine 2011; 10: 939-44.

9. Yuebing R. Application of isokinetic training in postoperative early rehabilitation for elbow fracture. J Qingdao University 2011; 15: 75-84.

10. Ruyun Y, Dingwen Q, Baoxin H, Xiufen H, Xieyuan J. Systematic rehabilitation of intra-knee fracture in perioperative period. J Chin J Rehabilitation Med 2013; 5: $425-37$. 


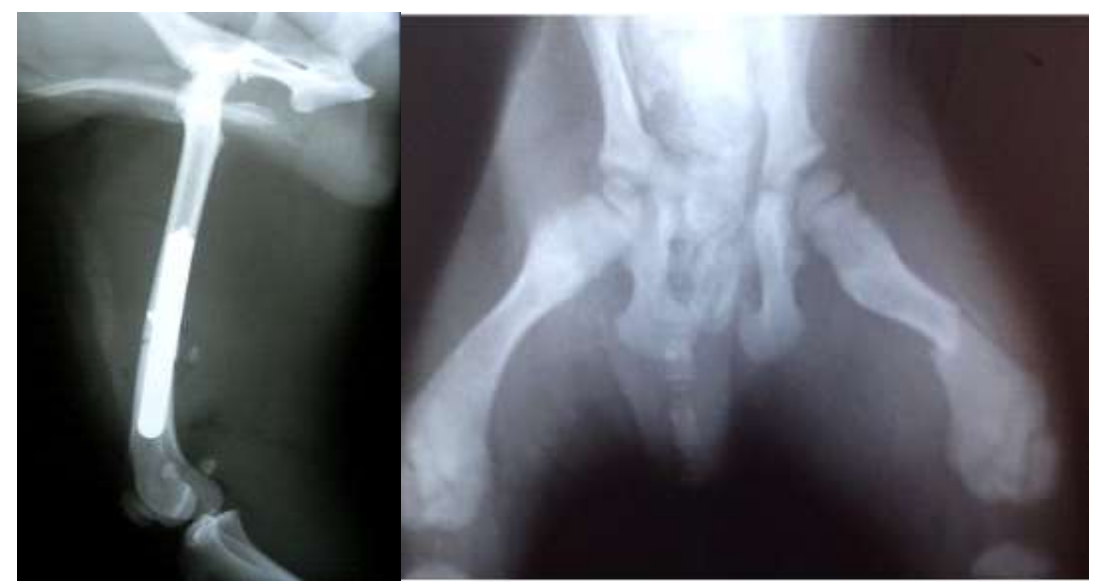

Fig. 1: The X ray Pictures of fracture.

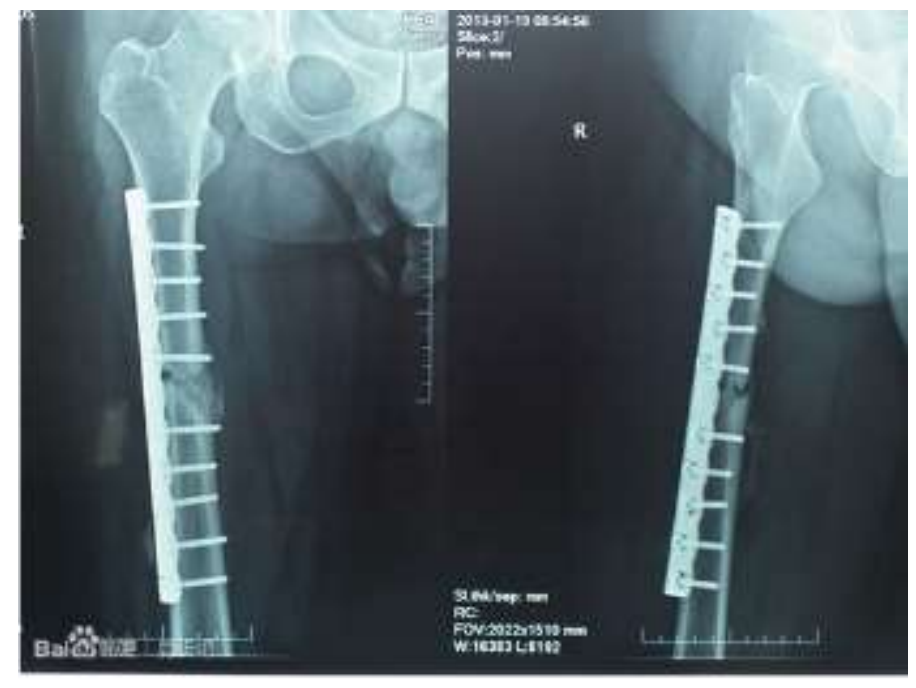

Fig. 2: The X ray Pictures of fracture treatment. 